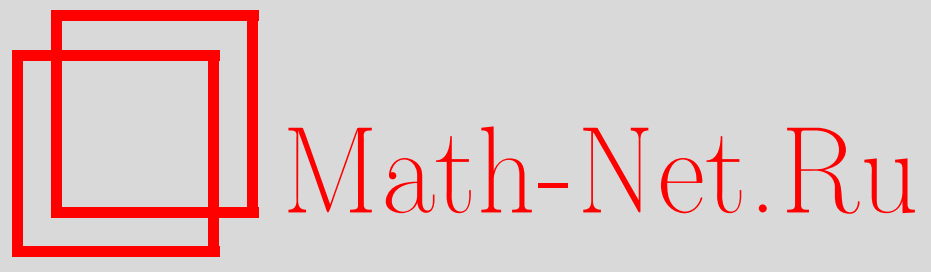

А. Д. Казанцев, Монотонное упрощение книжных представлений некоторых сателлитных узлов, УМH, 2010, том 65, выпуск 4, 195-196

DOI: https://doi.org/10.4213/rm9370

Использование Общероссийского математического портала Math-Net.Ru подразумевает, что вы прочитали и согласны с пользовательским соглашением http://www . mathnet.ru/rus/agreement

Параметры загрузки:

IP: 52.23 .180 .231

26 апреля 2023 г., 03:24:09

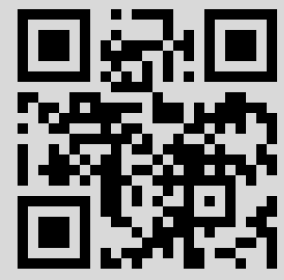




\title{
Монотонное упрощение книжных представлений некоторых сателлитных узлов
}

\author{
А. Д. Казанцев
}

В недавней работе [1] И. Дынникова было показано, что прямоугольную диаграмму тривиального узла можно привести к тривиальному виду с помощью монотонного упрощения. В той же работе показывается, что с помощью монотонного упрощения можно решить и задачу разложения узла или зацепления на простые неприводимые слагаемые. В настоящей работе изучается аналогичный вопрос: в некоторой специальной серии случаев прямоугольную диаграмму сателлитного узла можно монотонно упростить до такого вида, где структура несжимаемого тора (компаньона) из дополнения к этому узлу будет явно видна.

ОПредЕлениЕ. Сателлитным узлом в пространстве $S^{3}$ (или $\mathbb{R}^{3}$ ) называют узел $K$, в дополнении к которому содержится несжимаемый тор, не изотопный границе окрестности данного узла в пространстве $S^{3} \backslash K$ (соответственно $\mathbb{R}^{3} \backslash K$ ). По отношению к этому узлу $K$ тор или сердцевину тора мы будем называть компанъонами в зависимости от контекста.

В работе [1] явно описано, как по прямоугольной диаграмме узла получать соответствующее ей книжное представление узла и наоборот - как по книжному представлению получить прямоугольную диаграмму. Поэтому мы будем пользоваться эквивалентными в этом смысле языками прямоугольных диаграмм и книжных представлений, выбирая тот, который в данный момент удобнее.

ОпредЕлЕниЕ. Пусть $K_{\mathrm{b}}-$ книжное представление какого-то узла. Через $K_{\mathrm{r}}$ обозначим прямоугольную диаграмму, соответствующую этому книжному представлению. Будем говорить, что на прямоугольной диаграмме $K_{\mathrm{r}}$ непосредственно обнаружима структура компанъона, если все вертикальные и горизонтальные отрезки $K_{\mathrm{r}}$ находятся внутри замкнутой полосы, являющейся окрестностью другой прямоугольной диаграммы $r$. При этом книжное представление $r$ должно быть сердцевиной тора-компаньона, лежащего в пространстве $S^{3} \backslash K_{\mathrm{b}}$.

ОПРедЕЛЕНИЕ. Будем говорить, что структура тора-компаньона обнаружима $c$ помощъю монотонного упрощения на прямоугольной диаграмме $K_{\mathrm{r}}$ узла, если существует такая последовательность элементарных преобразований прямоугольных диаграмм узла, не увеличивающих сложность, что на итоговой диаграмме непосредственно обнаружима структура компаньона.

Упрощение до шахматной структуры. Всегда ли структура тора-компаньона обнаружима на прямоугольной диаграмме узла с помощью монотонного упрощения? Подобная задача, в которой узлы представлялись замкнутыми косами, а не прямоугольными диаграммами, изучалась ранее в работах Дж. Бирман и В. Менэско [2], [3]. Перенося рассуждения в этих работах на случай прямоугольных диаграмм, мы получаем, что любой несжимаемый тор, лежащий в дополнении к книжному представлению узла, может быть преобразован к виду, в котором слоение, высекаемое на нем страницами книжного представления, имеет шахматную структуру.

ОПредЕЛЕНИЕ. Будем говорить, что слоение, высекаемое страницами $\mathscr{D}_{t}=t \times$ $S_{\phi}^{1} \times I$, где $t-$ фиксировано, на поверхности, вложенной в $S^{3}=S_{\phi}^{1} * S_{\theta}^{1}$, имеет шахматную структуру, если выполняются условия:

1) отсутствуют замкнутые слои (особыми точками могут быть только седла);

2) поверхность может быть разбита на четырехугольники с вершинами в вершинах слоения (точки пересечения поверхности с окружностью $S_{\phi}^{1}$ ) и сторонами, идущими по слоям; в каждой вершине слоения сходятся ровно четыре четырехугольника;

3) каждый такой четырехугольник содержит ровно одну седловую точку; 
4) любые два седла, лежащие в соседних четырехугольниках, имеют разную ориентацию (подробнее см. [1]).

Как показала К. Нг [4], тор с шахматной структурой может быть устроен сложнее, чем первоначально утверждалось в работе [2], и потому неочевидно, что структура такого тора может быть видна на прямоугольной диаграмме узла.

Опишем серию торов, предложенную К. Нг. Поскольку книжное представление узла определено в пространстве $S^{3}$, являющемся джойном $S_{\phi}^{1} * S_{\theta}^{1}$, то для того, чтобы задать вложение поверхности $P$ в $S^{3}$, достаточно для каждого $t \in S_{\theta}^{1}$ задать ее пересечение со страницей $\mathscr{D}_{t}$. Такое вложение можно представлять себе как циклический поток картинок (параметризованный точками окружности $S_{\theta}^{1}$ ), являющихся пересечениями страниц с поверхностью. Для задания комбинаторного типа вложения достаточно указать пересечения поверхности с критическими страницами и по одному пересечению с некритическими страницами между ними.

ОПредЕлЕниЕ. Продавленной трубкой мы будем называть образ цилиндра $S^{1} \times I$ при вложении в сферу $S^{3}=S^{1} * S^{1}$, заданном последовательностью перестроек:

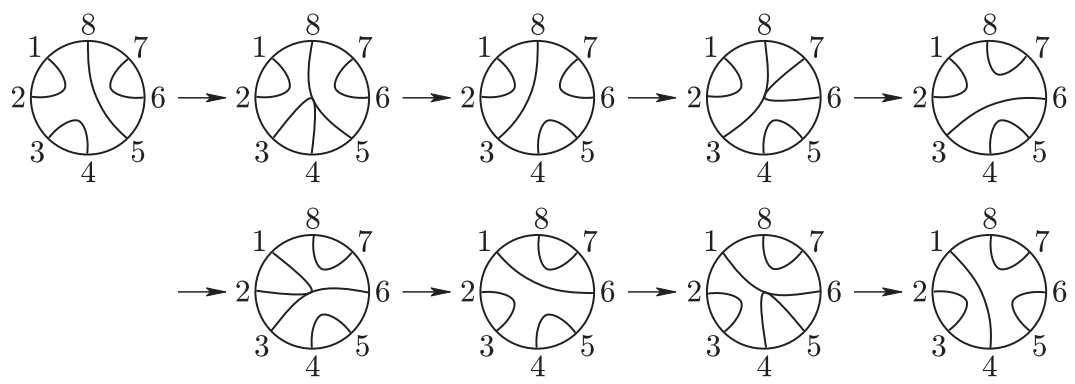

(Первая картинка в серии соответствует пересечению цилиндра со страницей $\mathscr{D}_{t_{i}}$, а последняя - со страницей $\mathscr{D}_{t_{j}}$. Здесь $\left(t_{i}, t_{j}\right)$ - некоторый отрезок окружности $S_{\theta}^{1}$.)

ОПредЕлениЕ. Продавленным тором мы будем называть образ двумерного тора $T^{2}=S^{1} \times S^{1}$ при вложении в 3-сферу: $\rho: T^{2} \hookrightarrow S^{3}=S^{1} * S^{1}$, если выполнены следующие условия: 1) тор $T^{2}$ склеен из цилиндров: $T^{2}=C_{1} \cup \cdots \cup C_{n}$ (цилиндры склеиваются в тор по своим основаниям); 2) образ каждого цилиндра $\rho\left(C_{i}\right)$ является продавленной трубкой.

УтвеРжДЕНИЕ. Продавленный тор обладает шахматной структурой.

Теорема. Пусть $K_{\mathrm{b}}$ - книжное представление некоторого сателлитного узла, $K_{\mathrm{r}}$ - прямоугольная диаграмма этого узла, соответствующая представлению $K_{\mathrm{b}}$, и $T$ - продавленный тор в дополнении $к$ узлу $\left(T \hookrightarrow S^{3} / K_{\mathrm{b}}\right)$, являющийся компаньоном. Тогда структура тора Т обнаружима на прямоугольной диаграмме узла с помощью монотонного упрощения.

ЗАмечание. Аналогичное утверждение для зацеплений неверно. Например, необнаружимые продавленные торы существуют на неупрощаемой прямоугольной диаграмме торического зацепления $(4,4)$.

\section{Список литературы}

[1] I. A. Dynnikov, Fund. Math, 190 (2006), 29-76. [2] J. S. Birman, W. W. Menasco, Topology, 33:3 (1994), 525-556. [3] J. S. Birman, W. W. Menasco, Topology, 37:1 (1998), 225. [4] K. Y. Ng, J. Knot Theory Ramifications, 7:2 (1998), 205-216.

А. Д. Казанцев (А. D. Kazantsev)

Московский государственный университет им. М. В. Ломоносова

E-mail: kaza57@gmail.com
Представлено И. А. Дынниковым Принято редколлегией 24.05 .2010 